\title{
Effect of atorvastatin on hard exudates in type 2 diabetic patients with normal lipid profile
}

\author{
Anubha Bhatti ${ }^{1, *}$, Subina Narang ${ }^{2}$ \\ ${ }^{\mathbf{1}}$ Assistant Professor, Dept. of Ophthalmology, Sri Guru Ram Das Institute of Medical Sciences \& Research, Amritsar, Punjab, \\ ${ }^{2}$ Associate Professor, Dept. of Ophthalmology, Government Medical College \& Hospital Chandigarh, Punjab, India
}

*Corresponding Author:

Email: scorpiodoc9@gmail.com

\begin{abstract}
Purpose: To evaluate the effect of Atorvastatin on hard exudates in diabetic retinopathy in type 2 diabetic patients with normal lipid profile.

Materials and Methods: Prospective study of 50 patients with clinically significant macular edema involving centre was done over 2 years (2015-16). We excluded patients with type 1 diabetes mellitus, patients with nephropathy/ heart disease, dyslipidemic patients, patients with laser treatment within 3 months of enrollment, patients with proliferative diabetic retinopathy requiring laser treatment. Detailed history was taken and thorough examination was done with $+90 \mathrm{D}$ lens for size and extent of clinically significant macular edema and grading of hard exudates. OCT was done for maximum retinal thickness. The outcome measures were visual acuity, change in grade of hard exudates and maximum retinal thickness. Change in maximum retinal thickness by $30 \%$ was considered as significant reduction of macular edema. Patient had minimum follow up of 6 months.

Results: Of 50 patients 34 were male and 16 female. Mean follow up period was $10.5 \pm 4.0$ months. Baseline mean visual acuity was $0.73 \pm 0.4 \log$ MAR and baseline mean MRT was $360.03 \pm 30.94 \mu$. Patients with grade 3 hard exudates were 6 , with grade 4 hard exudates were 28 and with grade 5 hard exudates were 16 at baseline. Final mean visual acuity was $0.76 \pm 0.4 \log$ MAR. Mean MRT at final visit was $284.6 \pm 48.3 \mu$. 34 patients $(68 \%)$ showed improvement by one grade and 2 patients showed improvement by two grade. The grade of hard exudates remained unchanged in 12 patients (24\%) and deteriorated in 2 patients. Conclusion: Atorvastatin leads to significant improvement in grade of hard exudates in clinically significant macular edema and decrease in maximum retinal thickness on OCT. However visual acuity status was not significant over short term follow up.
\end{abstract}

Keywords: Atorvastatin, Clinically significant macular edema, Hard exudates.

\section{Introduction}

Diabetic retinopathy (DR) \& diabetic macular edema are the leading cause of blindness in working age population in both developed and developing countries. ${ }^{1}$ According to a study done by Sankara Nethralaya in 2009, the prevalence of diabetic retinopathy in an urban Indian population with diabetes mellitus was $18 \% .^{2}$ Hyperlipidemia has been linked to the presence of retinal hard exudates in patients with DR and increases the risk of visual loss by $50 \%{ }^{3}$ The Early Treatment Diabetic Retinopathy Study (ETDRS) data suggested that lipid-lowering therapy may reduce hard exudates and microaneurysms \& helped in preserving vision in patients with clinically significant macular edema (CSME). ${ }^{4}$

Lipid lowering drugs like statins are the standard of care for CSME with dyslipidemia. Statins are 3hydroxy-3-methylglutaryl coenzyme A (HMG CoA) reductase inhibitors and they also have anti thrombotic, anti inflammatory and anti proliferative properties. Clinical studies have shown beneficial effect of statins on DR. In a study by Gordon et al pravastatin improved the appearance of hard exudates and microaneurysms. ${ }^{5}$ Another study reported improvement of visual acuity in diabetic patients with hypercholesterolemia using simvastatin. ${ }^{6}$ Later studies showed atorvastatin reduce the severity of hard exudates and microaneurysms in patients with diabetic macular edema. ${ }^{7,8}$ The role of statins in improvement of endothelial function and ocular blood flow has been emphasized. Atorvastatin improved retinal blood flow velocities in patients with background or proliferative DR. ${ }^{9}$

It was reported that intensive glycemic control and combination treatment of dyslipidemia reduced the rate of progression of DR. ${ }^{10}$ The mechanism, however, seemed to be related to intraretinal lipid transportation rather than serum lipid levels. However, to our knowledge, no study has evaluated the role of lipidlowering drugs in the management of clinically significant macular edema with normal lipid profile. In the present study, we evaluated the role of Atorvastatin in Clinically Significant Macular Edema (CSME) in type 2 diabetic patients with normal lipid profile.

\section{Materials and Methods}

This was a prospective randomised study including 50 metabolically stable diabetic (34 males \& 16 females) with CSME involving macular centre (20152016). We included eyes with non-proliferative diabetic retinopathy (NPDR) with CSME that presented to retina clinic of our institute which is a tertiary eye care centre. The study was approved by our institute ethical committee. The study included type 2 diabetic patients with CSME with normal lipid profile i.e. total cholesterol < 190mg\%, LDL < 115mg\%, HDL > $40 \mathrm{mg} \%$ and serum triglycerides $<180 \mathrm{mg} \%$ was taken as standard. We excluded patients with type 1 diabetes mellitus, patients with nephropathy/ heart disease, 
patients who had been subjected to pars plana vitrectomy for any reason, dyslipidemic patients, patients with laser treatment within 3 months of enrolment, patients with proliferative diabetic retinopathy requiring laser treatment.

Detailed history and thorough ocular examination including visual acuity by Snellen's chart, intraocular pressure by Goldmann applanation tonometer, slit lamp biomicroscopy, fundus evaluation was done with +90 D to look for the extent \& location of the hard exudates. Macular OCT was also done for size and extent of CSME. Grading of hard exudates was done as per ETDRS Report No.10. ${ }^{11}$ Atorvastatin in dosage of 10$20 \mathrm{mg}$ was started in consultation with internist. Patient had follow up at 1 month, at 3 months, at 6 months and thereafter. Patient had a minimum follow up of 6 months.

\section{Results}

The study included 50 patients of which 34 were male and 16 were female with type 2 diabetes with normal lipid profile. The Mean age \pm SD was $54.55 \pm 7.7$ years. Mean follow up period was $10.5 \pm 4.0$ months.

The outcome was evaluated in terms of visual acuity, change in grade of hard exudate and change in maximum retinal thickness. Visual acuity outcome was regarded as improvement (two lines gain in VA), stabilization (within two lines of initial VA) or deterioration (loss of two lines). Hard exudates status was regarded as improved, stable or worsened depending on any change in grade of hard exudates. Change in maximum retinal thickness by $30 \%$ was considered as significant reduction of macular edema.

Baseline mean visual acuity was $0.73 \pm 0.4 \mathrm{log}$ MAR and baseline mean MRT was 360.03 $\pm 30.94 \mu$. Final mean visual acuity was $0.76 \pm 0.4 \log$ MAR and mean MRT at final visit was $284.6 \pm 48.3 \mu$ (Table1). The change in visual acuity from baseline was not statistically significant as $\mathrm{p}$ value is 0.92 which is $>0.05$. The change in maximum retinal thickness from baseline was statistically significant ( $\mathrm{p}$ value $<0.001$ )

Patients with grade 3 hard exudates were $6(12 \%)$, with grade 4 hard exudates were $28(56 \%)$ and with grade 5 hard exudates were $16(32 \%)$ at baseline (Table 2). 34 patients $(68 \%)$ showed improvement by one grade (Fig. $1 \& 2$ ) and 2 patients showed improvement by two grade. The grade of hard exudates remained unchanged in 12 patients $(24 \%)$ and deteriorated in 2 patients (Table 3).

Table 1: Showing baseline $\&$ at final visit change of VA and maximum retinal thickness

\begin{tabular}{|l|c|c|c|}
\hline & Baseline & Final Visit & P value \\
\hline VA $(\log M A R)$ & $0.73 \pm 0.4$ & $0.76 \pm 0.4$ & $>0.05$ \\
\hline MRT $(\mu \mathrm{m})$ & $360.03 \pm 30.94$ & $284.6 \pm 48.3$ & $<0.001$ \\
\hline
\end{tabular}

Table 2: Showing grading of hard exudates at baseline

\begin{tabular}{|l|c|}
\hline Grading of Hard Exudates & Baseline (n) \\
\hline Grade 3 & $6(12 \%)$ \\
\hline Grade 4 & $28(56 \%)$ \\
\hline Grade 5 & $16(32 \%)$ \\
\hline
\end{tabular}

Table 3: Showing status of hard exudates at final visit

\begin{tabular}{|l|c|}
\hline Status of Hard Exudates & Final visit (n) \\
\hline Improved & 36 \\
\hline Stabilized & 12 \\
\hline Worsened & 2 \\
\hline
\end{tabular}

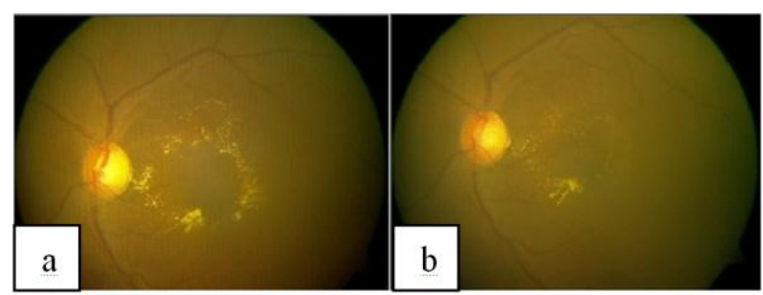

Fig.1: Fundus photograph $\mathrm{L} / \mathrm{E}$ of patient after 20 weeks atorvastatin therapy showing reduction in the macular edema as well as improvement in grade of hard exudates from grade 4 to grade 3 .

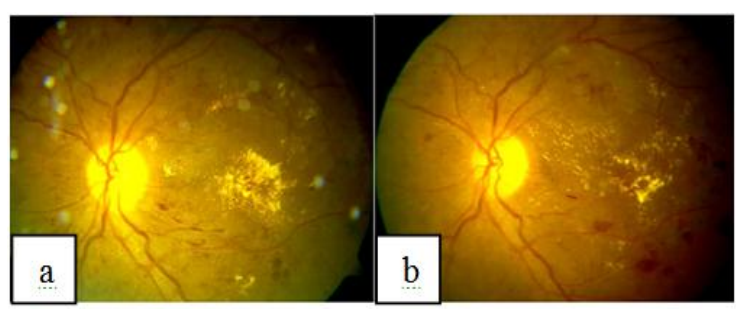

Fig. 2: Fundus photograph L/E of patient showing reduction in the macular edema as well as improvement in grade of hard exudates from grade 5 to grade 4 after 4 months of oral atorvastatin

\section{Discussion}

HMG Co A reductase inhibitors have been established as safe and efficacious drugs in management of diabetic retinopathy with dyslipidemia. Atorvastatin has beneficial effect in decreasing hard exudates severity and clinically significant macular edema in diabetics with dyslipidemia. ${ }^{12,13}$ The present study was to evaluate the role of atorvastatin in diabetics with normal lipid profile. Lipid exudation at the macula in diabetic patients is the result of increased vascular permeability and dysfunctional outer bloodretina barrier and retinal pigment epithelium. ${ }^{14}$ The Action to Control Cardiovascular Risk in Diabetes 
(ACCORD) eye studies demonstrated that intensive control of dyslipidemia using combined simvastatinfenofibrate treatment can significantly $(40 \%)$ delay the progression of DR in patients with Type 2 diabetes. ${ }^{15}$

A study by Gupta et al reported dramatic regression of hard exudates after correction of dyslipidemia in diabetics with severe hard exudates over a mean follow up of 18 weeks. $^{7}$ Thus, lipidlowering drugs preserve vision and reduce the risk of hard exudates in clinically-significant macular edema (CSME) in diabetics with an abnormal lipid profile. But their role in reducing CSME in diabetics with a normal lipid profile is not yet known. Our study showed that atorvastatin leads to significant decrease in grade of hard exudates \& also decreases maximum retinal thickness in type 2 diabetic patients with normal lipid profile.

Another study by Narang et al reported that atorvastatin does not affect treatment outcome in CSME over a short term follow-up. Results were based on visual acuity only. OCT and hard exudate grading was not done in that study as in our study. ${ }^{16}$

Michael Cusick et al showed histopathological evidence of regression of hard exudates and macular edema after reduction of elevated serum lipid levels in dyslipidemic patients. Now-a-days trends are shifting towards use of lipid lowering drugs as primary preventive measure for coronary artery disease in type II diabetics with normal lipid profile. ${ }^{17}$ Due to the histopathological similarity between diabetic retinopathy and coronary artery disease, atorvastatin could also have a role to play in diabetic retinopathy with normal lipid profile.

The results of our study suggest that lipid-lowering therapy in patients with diabetes may be a useful adjunct in the management of diabetic macular edema with severe hard exudates even in patients with normal lipid profile.

\section{Conclusion}

Atorvastatin leads to significant improvement in grade of hard exudates in clinically significant macular edema and decrease in maximum retinal thickness on OCT. However visual acuity status was not significant over short term follow up.

\section{Conflict of Interest: None}

\section{Source of Funding \& Support: Nil}

\section{References}

1. Thomas A C, Armando G A, Bernard Z. Diabetic Retinopathy \& diabetic macular edema: Pathophysiology, screening \& novel therapies. Diabetes Care. 2003;26:2653-2664.

2. Raman R, Rani PK, Reddi RS et al. Prevalence of diabetic retinopathy in India: Sankara Nethralaya Diabetic Retinopathy Epidemiology and Molecular Genetics Study report 2. Ophthalmology 2009;116:11-8.
3. Klein BE, Moss SE, Klein R, Surawicz TS. The Wisconsin Epidemiology Study of Diabetic Retinopathy. Relationship of serum cholesterol to retinopathy and hard exudates. Ophthalmology 1991;98:1261-1265.

4. Chew EY, Klein ML, Ferris FL et al. Association of elevated serum lipid levels with retinal hard exudate in diabetic retinopathy. Early Treatment Diabetic Retinopathy Study (ETDRS) Report 22. Arch Ophthalmol 1996;114:1079-1084.

5. Gordon B, Chang S, Kavanagh M, et al. The effects of lipid lowering on diabetic retinopathy. Am. J. Ophthalmol. 1991;112(4):385-391.

6. Sen K, Misra A, Kumar A, Pandey RM. Simvastatin retards progression of retinopathy in diabetic patients with hypercholesterolemia. Diabetes Res. Clin. Pract. 2002;56(1):1-11.

7. Gupta A, Gupta V, Thapar S, Bhansali A. Lipid lowering drug atorvastatin as an adjunct in the management of diabetic macular edema. Am J Ophthalmol 2004;137:675682.

8. Panagiotoglou TD, Ganotakis ES, Kymionis GD, et al. Atorvastatin for diabetic macular edema in patients with diabetes mellitus and elevated serum cholesterol. Ophthalmic Surg. Lasers Imaging. 2010;41(3):316-322.

9. Ozkiris A, Erkiliç K, Koç A, Mistik S. Effect of atorvastatin on ocular blood flow velocities in patients with diabetic retinopathy. Br J Ophthalmol 2007;91:6973.

10. Keech AC, Mitchell P, Summanen PA et al. Effect of fenofibrate on the need for laser treatment for diabetic retinopathy (FIELD study): a randomized controlled trial. Lancet. 2007;370(9600):1687-1697.

11. Early Treatment Diabetic Retinopathy Study Research Group. Early diabetic retinopathy from stereoscopic color fundus photographs - an extension of the Modified Airlie House Classification. ETDRS report 10. Ophthalmology 1991;98:786-806.

12. Comer GM, Thomas A (2004). Pharmacotherapy for diabetic retinopathy. Curr Opin Ophthalmol;15:508-518.

13. Le NA, Innis-Whitehouse W, Li X et al. Lipid and apoprotein levels and distribution in patients with hyperglyceridemia: effect of triglyceride reduction with atorvastatin. Metabolism 2000;49:167-177.

14. Ali TK, El-Remessy AB. Diabetic retinopathy: current management and experimental therapeutic targets. Pharmacotherapy. 2009;29(2):182-192.

15. Chew EY, Ambrosius WT, Davis MD, et al. Effects of medical therapies on retinopathy progression in type 2 diabetes. N. Engl. J. Med. 2010;363(3):233-244.

16. Narang S, Sood S, Kaur B et al. Atorvastatin in clinicallysignificant macular edema in diabetics with a normal lipid profile. Nepal J Ophthalmol 2012;4 (7):23-28.

17. Michael C, Cusick M, Chew EY et al. Histopathology and regression of retinal hard exudates in diabetic retinopathy after reduction of elevated serum lipid levels. Ophthalmology 2003;110:2126-2133. 